\title{
Reduccionismo Didáctico y Creencias de Profesores acerca del Teorema de Pitágoras
}

\author{
Didactical Reductionism and Teachers' Beliefs about the \\ Pythagorean Theorem
}

\author{
Aarón Víctor Reyes-Rodríguez* \\ Carlos Rondero-Guerrero** \\ Juan Alberto Acosta-Hernández ${ }^{* * *}$ \\ Marcos Campos-Nava ${ }^{* * * *}$ \\ Agustín Alfredo Torres-Rodríguez ${ }^{* * * * *}$
}

\begin{abstract}
Resumen
En este artículo reportamos los resultados de una investigación exploratoria, cuyo interés fue identificar cómo las creencias que sostienen profesores en servicio sobre el Teorema de Pitágoras, son indicadores de un reduccionismo didáctico relativo a este resultado matemático. Se aplicaron cuestionarios a cinco profesores de matemáticas que laboran en un bachillerato público y una entrevista semi-estructurada a un profesor quien imparte cursos de física y matemáticas en una carrera de ingeniería en una universidad pública. Los datos recolectados se analizaron con base en seis categorías de creencias. Un resultado relevante del trabajo es que los profesores conciben al Teorema de Pitágoras como un hecho aislado y no como un conocimiento integrado a una
\end{abstract}

\footnotetext{
* Doctor en Ciencias en Matemática Educativa por el Centro de Investigación y de Estudios Avanzados del IPN (CINVESTAV IPN). Profesor-Investigador en la Universidad Autónoma del Estado de Hidalgo (UAEH), Mineral de la Reforma, Hidalgo, México. Dirección postal: Universidad Autónoma del Estado de Hidalgo, Área Académica de Matemáticas y Física, Carretera Pachuca - Tulancingo Km 4.5, Col. Carboneras, CP 42184, Mineral de la Reforma, Hidalgo, México. E-mail: aaron.reyes.rdz@gmail.com

${ }^{* *}$ Doctor en Ciencias en Matemática Educativa por el Centro de Investigación y de Estudios Avanzados del IPN (CINVESTAV IPN). Profesor-Investigador en la Universidad Autónoma del Estado de Hidalgo (UAEH), Mineral de la Reforma, Hidalgo, México. Dirección postal: Universidad Autónoma del Estado de Hidalgo, Área Académica de Matemáticas y Física, Carretera Pachuca - Tulancingo Km 4.5, Col. Carboneras, CP 42184, Mineral de la Reforma, Hidalgo, México. E-mail: ronderocar@gmail.com

*** Doctor en Matemática Educativa por el Centro de Investigación en Ciencia Aplicada y Tecnología Avanzada (CICATA IPN) Profesor-Investigador en la Universidad Autónoma del Estado de Hidalgo (UAEH), Mineral de la Reforma, Hidalgo, México. Dirección postal: Universidad Autónoma del Estado de Hidalgo, Área Académica de Matemáticas y Física, Carretera Pachuca - Tulancingo Km 4.5, Col. Carboneras, CP 42184, Mineral de la Reforma, Hidalgo, México. E-mail: acostah@uaeh.edu.mx

**** Maestro en Ciencias en Matemáticas y su Didáctica por la Universidad Autónoma del Estado de Hidalgo (UAEH). Profesor-Investigador en la Universidad Autónoma del Estado de Hidalgo (UAEH), Mineral de la Reforma, Hidalgo, México. Dirección postal: Universidad Autónoma del Estado de Hidalgo, Área Académica de Matemáticas y Física, Carretera Pachuca - Tulancingo Km 4.5, Col. Carboneras, CP 42184, Mineral de la Reforma, Hidalgo, México. E-mail: mcampos@uaeh.edu.mx

***** Maestro en Ciencias en Matemáticas y su Didáctica por la Universidad Autónoma del Estado de Hidalgo (UAEH). Profesor de asignatura del Instituto Tecnológico de Atitalaquia, Atitalaquia, Hidalgo, México. Dirección postal: Instituto Tecnológico de Atitalaquia, Departamento de Ciencias Básicas, Av. Tecnológico nº 9, Col. Tezoquipa, CP 42970, Hidalgo, México. E-mail: aatr68@ hotmail.com
} 
red altamente estructurada de ideas que abarcan una amplia variedad de ramas de la matemática. Por tanto, resulta conveniente que los profesores reflexionen acerca de sus propias creencias y las implicaciones que tienen sobre su actividad docente.

Palabras clave: Creencias de profesores. Reduccionismo Didáctico. Educación Matemática. Teorema de Pitágoras. Decantamiento epistemológico.

\begin{abstract}
In this paper, we report the results of an exploratory research in which the interest was to identify how beliefs that support teachers in service about the Pythagorean Theorem are indicators of a didactical reductionism on this mathematical result. Questionnaires were applied to five public high school mathematics teachers and a semi-structured interview with a teacher who imparts courses of physics and mathematics in an engineering course at a public university. The collected data were analyzed based on six beliefs categories. One relevant result of the work is that teachers conceive the Pythagorean Theorem as an isolated fact and not as an integrated approach to a highly structured network of ideas, covering a wide variety of mathematics knowledge branches. However, it seems suitable that teachers think about their own beliefs and implications that those beliefs have in the teaching activity.
\end{abstract}

Keywords: Teacher's Beliefs. Didactical Reductionism. Mathematical Education. Pythagorean Theorem. Epistemological decantation.

\title{
1 Introducción
}

Las creencias de los profesores son un tema central en la investigación en educación matemática, debido a que la formulación de objetivos, la toma de decisiones y demás acciones didácticas que los docentes llevan a cabo en el salón de clase están orientadas, en gran medida, por tales creencias (BANDURA, 2009). Particularmente, se ha obtenido evidencia de que la actividad que los profesores desarrollan en el aula no se basa únicamente en su conocimiento disciplinar, epistemológico y didáctico, o en lineamientos pedagógicos plasmados en los programas de estudio y los libros de texto, sino que se apoya fuertemente en sus creencias respecto de tópicos o ideas específicas (GILL; FIVES, 2015). Es decir, las creencias de los profesores constituyen un referente que orienta la elección de las actividades que proponen a sus estudiantes, la organización del escenario de instrucción y los mecanismos de evaluación del aprendizaje (FRANCIS; RAPACKI; EKER, 2015).

La importancia de las creencias de los profesores para la didáctica de las matemáticas se refleja en el volumen significativo de investigación acerca de este constructo que se ha llevado a cabo durante las últimas décadas tanto desde el punto de vista teórico como del práctico (SHULMAN, 1986). Los trabajos de investigación acerca de las creencias se pueden clasificar en cuatro grandes áreas: (1) adquisición y cambio de las creencias, (2) estructura de las creencias, (3) efecto de las creencias y (4) contenido de las creencias (BAR-TAL, 1990). 
Dada la influencia que ejercen las creencias en la actividad de los docentes, es necesario entender cómo están estructuradas, cómo se manifiestan en contextos específicos (ERNEST, 1989) y cuál es su impacto en la selección, diseño o rediseño de tareas de instrucción, la organización de las actividades en el aula o la evaluación del aprendizaje; dado que el escenario de clase y los procesos de pensamiento involucrados en las creencias son componentes organizados funcionalmente en un sistema (NESPOR, 1985).

El conocimiento sobre las creencias de los profesores puede aportar información esencial para formular propuestas encaminadas a fortalecer su formación profesional y, en consecuencia, lograr mejoras en las prácticas de enseñanza así como en el aprendizaje de los estudiantes (PAJARES, 1992; FIVES; BUEHL, 2008). En los programas de formación docente es importante prestar especial atención a las actividades de instrucción, ya que una modificación en las creencias requiere de cambios en las acciones que un profesor lleva a cabo (PAJARES, 1992), con la finalidad de provocar desequilibrios y propiciar reajustes o modificaciones en las creencias que no se pueden lograr únicamente mediante la persuasión.

Básicamente, cualquier cosa que comparta una conexión directa o indirecta con las matemáticas puede considerarse como un objeto de creencias. Algunos objetos de creencias son abstractos, como la naturaleza del conocimiento matemático. Otros son más concretos, por ejemplo, el Teorema de Pitágoras (TÖRNER, 2003). De manera tal que cabe la pregunta de investigación ¿cuáles son las creencias que predominan en los profesores de matemáticas acerca del teorema de Piágoras? Se ha identificado en la literatura que analizar las creencias respecto a tópicos específicos puede ser de mayor utilidad que intentar determinar las creencias del profesor en general. Así, el presente trabajo tiene como objetivo proporcionar evidencias acerca de cómo las creencias de los profesores en torno al Teorema de Pitágoras (TP) son un indicador de un reduccionismo didáctico sobre este saber.

Se eligió centrar la atención en el TP porque se ha identificado como el teorema individual más importante de la matemática (BRONOWSKI, 2011; KAKU, 1995). Sin embargo, a pesar de su relevancia, consideramos que existe un reduccionismo didáctico en torno a este resultado, en la educación escolarizada, lo cual significa que los profesores no reconocen las relaciones estructurales entre conceptos que son útiles para desarrollar un entendimiento profundo del TP (HIEBERT et al., 1997), por lo que en las aulas prevalece una visión atomizada del conocimiento matemático, que no permite ver cómo el TP se integra en una red estructurada de ideas que abarca diferentes áreas de la disciplina, entre las que se destacan la teoría de números, el álgebra, la trigonometría, la geometría analítica o el cálculo. 
A la red de conceptos y significados asociados con el TP la denominamos Relación Pitagórica (RP). En este trabajo consideramos que el reduccionismo didáctico está sustentado en las creencias de los profesores, y que a su vez, estas creencias se adoptan acríticamente por los estudiantes, cuando internalizan las prácticas y visión del mundo matemático de sus profesores (NESPOR, 1985).

La Relación Pitagórica es una red de significados, representaciones, contextos de uso, conceptualizaciones e interpretaciones, que se han estructurado alrededor del TP, como resultado de una evolución histórica y epistemológica: ternas Pitagóricas $(n, m, k)$, es decir ternas de números naturales que satisfacen la relación $n^{2}+m^{2}=k^{2}$; relación entre áreas de cuadrados construidos sobre los lados de un triángulo rectángulo; la expresión $a^{2}+b^{2}=c^{2}$ referida a la idea de circularidad y que se expresa en la relación $\operatorname{sen}^{2} \theta+\cos ^{2} \theta=1$; una familia de lugares geométricos asociada con la ecuación $\left(x-x_{1}\right)^{2}+\left(y-y_{1}\right)^{2}=r^{2}$; distancia entre dos puntos $\left(x_{2}-x_{1}\right)^{2}+\left(y_{2}-y_{1}\right)^{2}=d^{2}$; relación entre cantidades infinitesimales $d x^{2}+d y^{2}=d l^{2}$ (triángulo característico de Leibniz); entre otras. A pesar de esta riqueza de significados, en la matemática escolar la RP se reduce a considerar, la expresión algebraica $a^{2}+b^{2}=c^{2}$, sin asociarla con los referentes antes mencionados.

El reduccionismo en la ciencia se presenta cuando se adoptan posturas restrictivas respecto a la naturaleza, construcción y constitución del conocimiento, por ejemplo, cuando se considera que el todo no es más que la suma de las partes que lo integran (PÉREZ-TAMAYO, 2011). Por otro lado, el reduccionismo didáctico se expresa en términos de una desarticulación conceptual entre los contenidos disciplinares que se abordan en un mismo nivel o entre los diferentes niveles educativos, en una simplificación conceptual excesiva, la prevalencia de la algoritmia sobre el entendimiento conceptual, falta de interacción entre los contextos de uso que dan significado a los conceptos, así como una carencia de referentes históricos y epistemológicos asociados al entendimiento de los saberes matemáticos (LEUNG; BOLITE-FRANT, 2015).

Generalmente, los elementos que constituyen la RP se presentan y abordan como temas independientes entre sí, tanto en los programas de estudio como en la práctica escolar. En este sentido, el aprendizaje se limita a los aspectos enunciativos del conocimiento, sin profundizar en los procesos de significación. Es decir, el conocimiento matemático escolar acerca del TP es, esencialmente, un conocimiento declarativo que se visualiza como un conjunto de hechos aislados (ANDERSON, 1996), dando lugar a un reduccionismo didáctico. De manera tal que, conocer cuáles son las creencias que sostienen los profesores respecto al 
TP y cómo están estructuradas, puede ayudar a caracterizar y entender algunas de las causas e implicaciones del reduccionismo didáctico en torno al TP, lo cual a su vez, podría aportar elementos para formular propuestas que permitan enfrentar esta problemática.

\section{Marco conceptual}

Una dificultad al realizar investigación sobre las creencias se encuentra en la falta de consistencia de lo que se entiende por este término (TÖRNER, 2003). Generalmente, no se define lo que son las creencias (COONEY; SHEALY; ARVOLD, 1998) o cada investigador propone su propia definición, de forma que en ocasiones las diferentes definiciones son incluso contradictorias (FURINGHETTI; PEHKONEN, 2003). A pesar de la falta de precisión terminológica, existe un consenso generalizado de que las creencias son construcciones mentales personales subjetivamente verdaderas, que tienen un profundo componente afectivo y que no pueden modificarse solamente mediante la persuasión (PAJARES, 1992; SCHOENFELD, 1998).

Las creencias son cierto tipo de conocimiento que no requiere de un consenso general o grupal (PAJARES, 1992). Por ejemplo, un profesor puede creer que los estudiantes obtienen malas calificaciones porque simplemente no tienen buenos hábitos de estudio, o que es suficiente enunciar una definición o un teorema para que los estudiantes lo entiendan. Las creencias se refieren a lo que alguien sostiene como verdadero, independientemente de si otras personas estén de acuerdo o no, o de que sepan que la idea es verdadera o no. Las creencias son representaciones internas a las cuales el poseedor les atribuye verdad, validez o aplicabilidad, usualmente son estables, altamente cognitivas y pueden estar fuertemente estructuradas (GOLDIN, 2003). En este sentido, las creencias son independientes de su validez social, por lo que desde una perspectiva epistemológica, las creencias son constructos individuales, mientras que el conocimiento es un constructo social (OP 'T EYNDE; DE CORTE; VERSCHAFFEL, 2003).

Para Schoenfeld (1998) las creencias son construcciones mentales que representan una codificación de las experiencias y los entendimientos de las personas, así que el sistema de creencias personal está constituido por la visión del mundo matemático que cada uno de nosotros sostiene (SCHOENFELD, 1985). Al igual que el conocimiento, las creencias están organizadas en conglomerados alrededor de situaciones y contextos específicos, lo cual quiere 
decir que aquello que escuchamos, percibimos y comprendemos de una cierta situación puede que solamente sea aceptado como verdadero en ciertos contextos (BOGDAN, 1986).

Cuando se adopta una nueva creencia, ésta automáticamente se integra a una estructura de conocimiento subjetivo que constituye el sistema personal de creencias (FURINGUETTI; PEHKONEN, 2003). A partir de su incorporación a este sistema, la nueva creencia filtra y afecta, de forma determinante, el procesamiento de nueva información. Con el tiempo y el uso las creencias se conectan con más elementos en la red volviéndose más robustas, y por tanto más difíciles de modificar. En este sentido, a pesar de que el aprendizaje de un nuevo saber debe sustentarse en conocimientos previos; en ocasiones, este conocimiento viejo se convierte en un obstáculo para la construcción del nuevo conocimiento, aunque constituya un antecedente necesario. Así, que la superación de estos obstáculos frecuentemente es una parte importante en la construcción de significados para los nuevos conocimientos (BALACHEFF, 1990; BACHELARD, 2000).

\section{Metodología}

La presente investigación es de carácter exploratorio y cualitativo. De manera tal que la recolección de datos se llevó a cabo mediante un cuestionario de 12 preguntas de respuesta abierta. Al respecto, tanto Montoro (2014) como Gil y Rico (2003) sugieren que este tipo de cuestionarios se pueden aplicar para recoger las creencias y/o concepciones de los profesores sobre aspectos diversos de la enseñanza y el aprendizaje.

En nuestro caso, el cuestionario fue diseñado para indagar acerca de las creencias sobre el TP que sostienen profesores de matemáticas y, además de algunos de los saberes relacionados con el mismo. Las preguntas se elaboraron para obtener información sobre cuatro categorías acerca de las creencias, previamente identificadas como relevantes en lo que corresponde al objeto estudiado que es el TP. Las cuales son: (i) el enunciado del teorema, (ii) la importancia de justificar el resultado, (iii) la relevancia de los aspectos históricos y epistemológicos del teorema dentro de la matemática y (iv) su relación con otros saberes. Dado que las fuentes de información de este trabajo fueron cuestionarios y entrevistas, y no observaciones de clase, el análisis se enfocó en las creencias profesadas de los docentes (SCHOENFELD, 1998), es decir, aquellas creencias expresadas por ellos verbalmente o por escrito. 
El cuestionario se aplicó en el primer semestre de 2015 a una muestra no estadística, de cinco profesores voluntarios (identificados con un seudónimo), quienes trabajan en una escuela preparatoria pública de México. Los cinco participantes son ingenieros industriales y cuatro de ellos cursaron una maestría en enseñanza de las matemáticas o tecnología educativa. Al momento de la aplicación del cuestionario los profesores contaban con una experiencia docente de entre 13 y 32 años. También se realizó una entrevista semi-estructurada a un profesor de matemáticas y física, egresado de una licenciatura en matemáticas aplicadas y una maestría en matemáticas, que imparte cursos en carreras de ingeniería en una universidad pública desde hace tres años. Las preguntas que orientaron la entrevista se enfocaron a obtener información respecto de las creencias acerca del Teorema de Pitágoras y la percepción sobre los conocimientos que poseen los estudiantes a los que imparte clase, respecto a este tema.

Como señalan Gil y Rico (2003), los cuestionarios abiertos se pueden complementar con una entrevista semi-estructurada para contrastar los datos obtenidos. En forma tal que la entrevista tuvo la finalidad de contrastar las creencias de este profesor con las expresadas por los otros profesores en los cuestionarios abiertos. Adicionalmente en la entrevista, el profesor menciona algunas de las creencias de sus estudiantes, las que a su vez, al menos en parte, se identifican como un legado de lo aprendido en las aulas de bachillerato y que aún se expresan siendo estudiantes universitarios de ingeniería.

\section{Análisis de las creencias relacionadas con el Teorema de Pitágoras}

El análisis de los resultados se enfocó en dos aspectos principales, determinar cuáles son las creencias profesadas por los docentes en torno al TP, y cómo estas creencias son un indicador de un reduccionismo didáctico. Suponemos que si un profesor no logra identificar al TP como parte de una estructura de saberes, o no es capaz de ver cómo los saberes relacionados con el TP se integran en una red, es muy probable que, en su práctica docente, no considere la gran riqueza conceptual subyacente en este resultado matemático, incluyendo la identificación del TP como un conocimiento ancestral, parte de nuestra herencia cultural. Las creencias de los profesores se clasificaron en seis categorías que emergieron del análisis mismo de los datos obtenidos (GIL; RICO, 2003), reestructurando las cuatro categorías iniciales, quedando finalmente agrupadas como sigue: i) enunciados del TP; ii) formas de 
justificación; iii) aspectos históricos y epistemológicos; iv) contextos de uso; v) relevancia y vi) generalización o extensión.

(i) Creencias sobre los enunciados. La indicación a los profesores fue que enunciaran el TP, siendo la forma más común:

En un triángulo rectángulo, la hipotenusa al cuadrado es igual a la suma del cuadrado de los catetos

(Enunciado de los profesores, 2015).

Sin embargo, en algunos casos, los profesores no hicieron referencia al triángulo rectángulo y mencionaron que el enunciado del TP es $c^{2}=a^{2}+b^{2}$, sin dar mayor explicación, lo cual es muestra de una simplificación conceptual excesiva, o la falta de entendimiento de que un teorema es un enunciado condicional que debe ser probado. En este caso, el reduccionismo didáctico se expresa al considerar al TP como una fórmula o una ecuación, sin que intervengan otros elementos de reflexión, como la condición de que las variables se refieren a las longitudes de los lados de un triángulo rectángulo. Lo anterior concuerda con lo mencionado por el profesor universitario al preguntarle sobre los conocimientos de sus estudiantes respecto al TP:

[Los estudiantes] conocen el nombre del teorema e incluso lo llegan a enunciar, pero no lo saben interpretar, además para muchos estudiantes, enunciar el teorema se reduce a la expresión algebraica $a^{2}+b^{2}=c^{2}$

(Federico, 2015).

Si los profesores tienen la creencia que el TP es solamente una ecuación, esta creencia se verá reflejada en el conocimiento que poseen los estudiantes egresados del bachillerato.

(ii) Creencias sobre las formas de justificación. Al cuestionarles sobre cómo podrían justificar que el TP es cierto, la mayoría de los profesores expresaron que por medio de la comparación entre áreas:

la demostración usual o común es a través de la obtención de áreas con base a las longitudes tanto de los catetos como de la hipotenusa

(Enunciado de los profesores, 2015).

O propusieron el uso de casos particulares:

sustituyendo el valor de los lados de cualquier triángulo rectángulo y comprobando el Teorema

(Enunciado de los profesores, 2015).

$\mathrm{Al}$ respecto, un profesor utilizó únicamente el triángulo de lados $(3,4,5)$ para verificar la equivalencia de áreas, dividiendo en cuadrados unitarios los cuadrados construidos sobre los catetos e hipotenusa. Esto también es muestra de un reduccionismo didáctico, ya que es común encontrar profesores que no cuestionan los resultados matemáticos, y la justificación 
de los mismos se queda en el ámbito de lo particular. Mientras que la investigación en educación matemática y propuestas curriculares de carácter internacional promueven que los procesos de justificación y argumentación, así como la generalización de resultados sean los ejes de la formación matemática de los estudiantes (NCTM, 2000).

El profesor universitario mencionó que existen muchas demostraciones de diferentes tipos, y que usualmente él utiliza en sus cursos una de tipo geométrico, comentó que:

la mayoría de los estudiantes se sorprenden de que exista forma de demostrar o justificar la validez de un resultado matemático, dado que ellos están acostumbrados a no discutir la validez de las afirmaciones que el profesor hace

(Federico, 2015).

(Considerar a la matemática como un conjunto de resultados dados que son incuestionables y por tanto no se requiere de proporcionar argumentos para fundamentar su validez, es otra manifestación de reduccionismo didáctico).

(iii) Creencias acerca de aspectos históricos y epistemológicos. La pregunta correspondiente a esta categoría fue ¿qué es lo que conoce desde el punto de vista histórico acerca del Teorema de Pitágoras? Aunque la pregunta se refiere al aspecto histórico, a partir de los registros de sus respuestas podría llegarse a identificar algunos de carácter epistemológico. Un profesor contestó que:

se aplicó por primera vez en la construcción de la pirámide de Kefrén en Egipto, y este teorema fue descubierto por la escuela pitagórica

(Cuauhtémoc, 2015).

Y uno más respondió, categóricamente:

[Conozco a] su creador: Pitágoras

(Benito, 2015).

Las respuestas de los profesores de una u otra forma manifiestan su creencia de que los antecedentes históricos y epistemológicos no son relevantes en la construcción del saber matemático, además de sostener ideas que son erróneas, por ejemplo que el TP fue descubierto por los pitagóricos, cuando la contribución de esta escuela fue generalizar y demostrar el resultado. Las respuestas de los profesores de bachillerato contrastan con la respuesta del profesor universitario quien expresó conocer poco sobre los aspectos históricos:

sin embargo considero que es importante abordar la parte histórica con los estudiantes ya que podría ser un elemento motivador (Federico, 2015).

(iv) Creencias respecto a los contextos de uso. ¿Conoce algunas aplicaciones del Teorema de Pitágoras? ¿En qué temas de las asignaturas que imparte hace uso del Teorema de 
Pitágoras? Los profesores consideran que las aplicaciones del TP se relacionan con el cálculo de distancias particularmente cuando no es factible realizar medidas directas:

[El Teorema de Pitágoras se aplica en] pintura (arte), construcción. Tanto arquitectos como ingenieros lo utilizan para la construcción de rampas, con o sin escalones

(Enunciado de los profesores, 2015).

O para

Calcular áreas, perímetros, longitudes

(Enunciado de los profesores, 2015).

Todos los profesores expresaron que el TP tiene aplicaciones en trigonometría (para el cálculo de longitudes), mecánica (para calcular la norma de vectores), electricidad y magnetismo (fuerzas resultantes) y cálculo integral (integración por sustitución trigonométrica). En las respuestas se evidencia la creencia de que el TP se utiliza en diversos contextos, pero sólo como una fórmula que permite calcular distancias, sin que se explicitaran las articulaciones conceptuales entre esos diversos temas. Lo anterior contrasta también con el profesor universitario, quien expresó dificultades para identificar que en mecánica el TP aparece en el tema de vectores, en geometría analítica con distancia entre puntos, ecuación de una circunferencia, o en problemas de electromagnetismo y óptica geométrica para calcular distancias. Se conjetura que la experiencia laboral como profesor es un factor que puede modificar algunas las creencias de los profesores, sobre todo en la categoría de contextos de uso, ya que los profesores identificaron diversos contextos del TP. En este caso el reduccionismo didáctico se manifiesta por medio de que el TP es una fórmula para calcular distancias que se emplea en diversos contextos, sin que se contrasten y expliciten los significados inherentes a cada uno de los contextos.

(v) Creencias sobre su relevancia. Los profesores de matemáticas consideran que las diferentes áreas de la matemática no tienen relación entre sí, por lo cual el reduccionismo didáctico en este sentido se presenta al exponer contenidos fragmentados sin un manifiesto interés por articularlos. En este sentido, los profesores reconocen que el TP es importante porque permite calcular distancias que no se pueden medir directamente, lo cual es relevante en disciplinas como astronomía e ingeniería. También hicieron referencia a otras aplicaciones de tipo práctico, como calcular la longitud de un cable que sujeta a un poste o para la

orientación, [para] conocer distancias, orientaciones geográficas

(Enunciado de los profesores, 2015). 
Lo que da evidencia de que los profesores no identifican al TP como uno de los teoremas más importantes de la matemática, ni que es fundamental para describir el espacio en el que vivimos.

Por otro lado, el profesor universitario mencionó que existe una estrecha relación del TP con la trigonometría, pero que casi nunca se demuestra la identidad $\operatorname{sen}^{2} \theta+\cos ^{2} \theta=1$, por medio del círculo unitario o utilizando triángulos rectángulos y el TP, lo atribuye a que el temario o programa de estudios presenta los temas por separado sin relación unos con otros; lo cual es muestra del reduccionismo didáctico motivado por la creencia de que las diferentes áreas de la matemática no guardan una estrecha relación entre sí, visión que se promueve tanto en los programas de estudio como en los libros de texto.

(vi) Creencias respecto a la generalización o extensión. ¿Es posible generalizar el Teorema de Pitágoras? Un profesor respondió que sí, es posible, pero no explicó cómo se lleva a cabo tal generalización:

En la ley de los cosenos como el Teorema de Pitágoras extendido (Cuauhtémoc, 2015)

sin embargo, la mayoría de los profesores respondió que no es posible una generalización:

Solo es aplicable para los triángulos rectángulos

(Enunciado de los profesores, 2015).

Lo que permite concluir que la mayor parte de los profesores no entienden lo que significa generalizar un resultado matemático. Cabe resaltar que otro tipo de generalización sobre el TP es cuando se plantea una relación de igualdad entre áreas para figuras que no son necesariamente cuadrados, por ejemplo semicircunferencias cuyo diámetro son los lados de un triángulo rectángulo o polígonos regulares que se construyen a partir de considerar uno de sus lados, los catetos e hipotenusa de un triángulo rectángulo; en este sentido, también se puede remarcar que los profesores sostienen la creencia de que el teorema es válido sólo para comparación entre áreas de cuadrados, al no mencionar como una variante del enunciado ésta posible generalización del TP.

Se preguntó al profesor universitario si ha discutido con los estudiantes que la relación entre las áreas de los cuadrados construidos sobre los lados de un triángulo rectángulo también es válida si se construyen otro tipo de figuras semejantes sobre esos mismos lados:

he visto antes esas relaciones, pero no las he trabajado en mis clases, y considero que a los estudiantes les podría resultar interesante dado que tradicionalmente sólo se trabaja con cuadrados

(Federico, 2015). 
En este sentido, la generalización es un medio para articular ideas matemáticas, y los profesores participantes no le toman relevancia a este hecho, que además se promueve desde la estructura de los programas de estudio y libros de texto, donde se ha verificado que no se hace mención de este tipo de generalizaciones del TP.

\section{Conclusiones}

En esta aproximación inicial a la relación entre las creencias de profesores acerca del TP y el reduccionismo didáctico, queda de manifiesto que este resultado no se encuentra articulado fluidamente con otros conceptos, excepto, en algunos casos, con la idea de distancia. También se observó que los profesores otorgan poca importancia a la justificación de resultados matemáticos, restringiendo esta actividad a la identificación de algunos casos particulares. Por ejemplo, al utilizar el triángulo de lados $(3,4,5)$ para sustentar la validez del Teorema de Pitágoras. La representación predominante del TP es en el contexto geométrico, aunque en algunos casos la imagen mental del TP se restringe a una representación algebraica.

Es patente también el poco interés de los profesores por la génesis y evolución histórica de este saber, limitándose en la mayoría de los casos a mencionar que su creador es el matemático griego Pitágoras, y sólo en algunos casos aislados se hizo mención de que este resultado era conocido ya por la cultura egipcia. La mayoría de los profesores hicieron referencia a la transversalidad de este teorema en la matemática, al contextualizarlo en áreas tales como trigonometría, geometría analítica y cálculo, además de algunas áreas de la física como en mecánica clásica, electrostática y óptica geométrica; además de identificar diversos usos potenciales dentro y fuera de la matemática como en el diseño de rampas en estacionamientos, y el cálculo de distancias que no se pueden medir directamente.

Observamos que en el devenir del tiempo, el TP ha sufrido un reduccionismo didáctico que se expresa como una especie de decantamiento epistemológico, reflejado en las respuestas de los profesores en las que el TP se reduce a la expresión $a^{2}+b^{2}=c^{2}$, la cual conlleva una carencia de significados y relaciones, que son base del entendimiento matemático. Este decantamiento es análogo al fenómeno de la invisibilidad de las tecnologías identificado por Moreno-Armella y Sriraman (2005). En este caso, el TP al ser una de las herramientas fundamentales de la matemática, se ha incorporado de forma estrecha al saber matemático, aunque durante la educación escolarizada se reproduce ese carácter de invisibilidad del saber, de manera que éste se incorpora acríticamente a la cultura matemática como una serie de 
creencias socialmente compartidas, despojando al TP de sus elementos conceptuales históricos y epistemológicos, los cuales son de gran trascendencia en la construcción del conocimiento matemático. Reflexionar respecto al tema del reduccionismo didáctico, asociado a su decantamiento epistemológico, es importante porque la instrucción matemática debiera proporcionar a los estudiantes un sentido holístico de los conceptos, de su alcance, potencia, usos e historia, adecuado a la experiencia y nivel de entendimiento particular de los estudiantes (SCHOENFELD, 1998).

Otro aspecto interesante de remarcar, es el hecho de que ningún profesor hizo referencia al recíproco del teorema, es decir, si la suma de las áreas de los cuadrados de dos lados de un triángulo, es igual al área del cuadrado del tercer lado, entonces el triángulo es rectángulo, o al hecho de que la longitud de la hipotenusa es mayor que la longitud de cualquiera de los catetos, en forma tal que algunos profesores tienen la creencia de que el enunciado verbal, o expresado algebraicamente, es suficiente para que sus estudiantes entiendan el TP, según sus propias creencias, dejando de lado los diferentes significados o personificaciones de la RP (ABRAHAMSON, 2009).

Resulta conveniente remarcar que el reduccionismo didáctico se manifestó a través de las creencias de los profesores en diferentes formas. Por ejemplo, cuando la justificación se restringe a la consideración de casos particulares y no se le otorga importancia a la generalización de resultados o cuando no se le atribuye relevancia a los aspectos históricos en la construcción del conocimiento matemático. En cuyo caso se pierde la oportunidad de aprovechar la génesis epistemológica como un medio para promover el entendimiento de los conceptos matemáticos, tratando de ése modo de revertir, al menos en parte, lo que hemos denominado decantamiento epistemológico.

Con base en lo expresado en este artículo se pudo observar que en el ámbito disciplinar el TP tiene un papel preponderante en la construcción del conocimiento matemático, mientras que en el ámbito didáctico actual tiene un estatus limitado, ya que se percibe como un hecho matemático asociado esencialmente con el cálculo de distancias. Conjeturamos que este contraste radica en la conformación de un sistema de creencias socialmente compartido, que se va trasmitiendo de una generación a otra, el cual ha propiciado que el TP sufra un cierto decantamiento epistemológico, hasta quedar prácticamente reducido a un simple enunciado o expresión algebraica, sin atender a la amplitud de conceptos asociados a sus significados inherentes. 
Las creencias matemáticas tienen asociados aspectos de contenido histórico y epistemológico. En el caso del TP, las creencias generalmente se restringen a una relación directa a la figura de Pitágoras, quien, sin duda, es uno de los grandes matemáticos de la historia, y debido al peso que conlleva el nombre asociado al resultado matemático, ésta asociación se consolida como una creencia prototípica que se vuelve incuestionable para la didáctica, ocasionando una tendencia a considerar que la finalidad del aprendizaje es la memorización de enunciados y la repetición de procedimientos, más que el desarrollo de procesos de argumentación, demostración, validación y articulación de conceptos y resultados.

En un aula de clase donde está presente un reduccionismo didáctico es difícil que se promueva el entendimiento de las ideas matemáticas, ya que éste depende, en gran medida, de la construcción de conexiones robustas entre previos y nuevos conocimientos. Este carácter atemporal e impersonal de la matemática escolar (D’AMORE, 2004), es un reflejo de las creencias de los profesores de bachillerato. Ocurre, entonces, que se presenta una cierta forma de perpetuidad de las creencias de una a otra generación, lo que impide al sistema escolar renovarse. Resulta por tanto importante que el profesor reflexione acerca de sus propias creencias y el impacto que éstas tienen sobre su actividad docente. Por otra parte, los programas de formación y actualización de profesores deberían de promover actividades que les permitan cuestionar sus creencias sobre sus conocimientos disciplinares y didácticos.

\section{Referencias}

ABRAHAMSON, D. Embodied design: constructing means for constructing meaning. Educational Studies in Mathematics, Amsterdam, v. 70, p. 27-47, ene. 2009.

ANDERSON, J. The architecture of cognition. 1. ed. New York: Psychology Press, 1996. 345 p.

BACHELARD, G. La formación del espíritu científico. 23. ed. Traducción de J. Babini. Ciudad de México: Siglo XXI, 2000. 302 p.

BALACHEFF, N. Towards a problématique for research on mathematics teaching. Journal for Research in Mathematics Education, Reston, v. 21, n. 4, p. 258-272, jul. 1990.

BANDURA, A. Self-efficacy in changing societies. 1. ed. Cambridge: Cambridge University Press, 2009. $334 \mathrm{p}$.

BAR-TAL, D. Group Beliefs: A conception for analyzing group structure, processes, and behavior. 1. ed. New York: Springer, 1990. 140 p.

BOGDAN, R. J. The importance of belief. En: BOGDAN, R.J. (Ed.). Belief: Form, content, and function. New York, NY: Oxford University Press, 1986. p. 1-16. 
BRONOWSKI, J. The ascent of man. 4. ed. London: BBC Books, 2011. 332 p.

COONEY, T. J.; SHEALY, B. E.; ARVOLD, B. Conceptualizing belief structures of preservice secondary mathematics teachers. Journal for Research in Mathematics Education, Reston, v. 29, n. 3, p. 306-333, may.1998.

D’AMORE, B. El papel de la epistemología en la formación de profesores de Matemáticas en la escuela secundaria. Epsilon, Revista de la Sociedad Andaluza de Educación Matemática, Sevilla, n. 60 , p. 413-434, sep./dic. 2004.

ERNEST, P. The knowledge, beliefs, and attitudes of the mathematics teacher: A model. Journal of Education for Teaching, Londres, v. 15, n. 1, p. 13-33, ene./abr.1989.

FIVES, H.; BUEHL, M. M. What do teachers believe? Developing a framework for examining beliefs about teachers' knowledge and ability. Contemporary Educational Psychology, Amsterdam, v. 33, n. 2, p. 134-176, abr. 2008.

FRANCIS D. C.; RAPACKI, L.; EKER, A. The individual, the context, and practice: A review of the research on teachers' beliefs related to mathematics, En: FIVES, H.; GILL, M.G. (Ed.). International handbook of research on teachers' beliefs. New York: Routledge, 2015. p. 336-352.

FURINGHETTI, F.; PEHKONEN, E. Rethinking characterization of beliefs, En: LEDER, G.C.; PEHKONEN, E.; TÖRNER, G. (Ed.). Beliefs: A Hidden variable in mathematics education? New York: Kluwer Academic Publisher, 2003. p. 39-57.

GIL, F.; RICO, L. Concepciones y creencias del profesorado de secundaria sobre enseñanza y aprendizaje de las matemáticas. Enseñanza de las Ciencias, Barcelona, v. 21. n.1. p.27-47, ene./abr. 2003.

GILL, M. G.; FIVES, H. Introduction. En: FIVES, H.; GILL, M.G. (Eds.). International handbook of research on teachers' beliefs, New York: Routledge, 2015. p. 1-10.

GOLDIN, G. A. Affect, meta-affect, and mathematical belief structures. En: LEDER, G.C.; PEHKONEN, E.; TÖRNER, G. (Ed.). Beliefs: A Hidden variable in mathematics education? New York: Kluwer Academic Publisher, 2003. p. 59-72.

HIEBERT, J. et al. Making sense: teaching and learning mathematics with understanding. Portsmouth, NH: Heinemann, 1997.

KAKU, M. Hyperspace: A scientific odyssey through parallel universes, time wraps, and the tenth dimension. 1. ed. New York: Anchor Books, 1995. 359 p.

LEUNG, A.; BOLITE-FRANT, J. Designing mathematics tasks: The role of tools. En: WATSON, A.; OHTANI, M. (Ed.). Task design in mathematics education: An ICMI study 22. Cham: Springer, 2015. p. 191-222.

MONTORO, A. Motivación y matemáticas: Experiencias de flujo en estudiantes de maestro de educación primaria. 2014. 277 h. Tesis (Doctorado en Investigación Didáctica) - Departamento de Educación, Universidad de Almería, Almería, 2014

MORENO-ARMELLA, L.; SRIRAMAN, B. The articulation of symbols and mediation in mathematics education, ZDM Mathematics Education, Amsterdam, v. 37, n. 6, p. 476-486, dic. 2005. 
NATIONAL COUNCIL OF TEACHERS OF MATHEMATICS [NCTM]. Principles and Standards for School Mathematics. 1. ed. Reston, VA: NCTM, 2000. 402 p.

NESPOR, J. The role of beliefs in the practice of teaching: Final report of the teacher beliefs study. 1. ed. Austin, Texas: Texas University, Research and Development Center for Teacher Education, 1985. $224 \mathrm{p}$.

OP'T EYNDE, P.; DE CORTE, E.; VERSCHAFFEL, L. Framing students' mathematics-related beliefs, En: LEDER, G.C.; PEHKONEN, E.; TÖRNER,G. (Ed.). Beliefs: A hidden variable in mathematics education? New York: Kluwer Academic, 2003. p. 13-38.

PAJARES, M. F. Teachers' beliefs and educational research: Cleaning up a messy construct. Review of Educational Research, Thousand Oaks, v. 62, p. 307-332, sep. 1992.

PÉREZ-TAMAYO, R. Acerca de Minerva. 3. ed. Ciudad de México: Fondo de Cultura Económica, 2011. $206 \mathrm{p}$.

SCHOENFELD, A. H. Mathematical Problem Solving. 1. ed. Orlando, FL: Academic Press, 1985. $402 \mathrm{p}$.

SCHOENFELD, A. H. Toward a theory of teaching-in-context. Issues in Education, Amsterdam, v. 4, n. 1, p. 1-94, ene./jul. 1998.

SHULMAN, L. S. Those who understand: Knowledge growth in teaching. Educational Researcher, Thousand Oaks, v. 15, n. 2, p. 4-14, feb.1986.

TÖRNER, G. Mathematical beliefs - a search for a common ground: some theoretical considerations on structuring beliefs, some research questions, and some phenomenological observations. En: LEDER, G.C.; PEHKONEN, E.; TÖRNER, G. (Ed.). Beliefs: A hidden variable in mathematics education? New York: Kluwer Academic Publishers, 2003. p. 73-94.

Submetido em 2 de Maio de 2016. Aprovado em 2 de Março de 2017. 